\title{
RESULTS FROM FIVE YEARS OF MONITORING OF THE EINSTEIN CROSS WITH NOT
}

R. ØSTENSEN

Auroral Observatory, University of Tromsø

N-9037 Tromsø, Norway

S. REFSDAL

Hamburg Sternwarte

Gojenbergsweg 112, D-21029 Hamburg, Germany

R. STABELL

Institute of Theoretical Astrophysics, University of Oslo

Blindern, N-0315 Oslo, Norway

AND

J. TEUBER

Copenhagen University Observatory

Brorfelde, DK-4340 Tølløse, Denmark

After some preliminary observations in 1990, a program was started in 1991 at the Nordic Optical Telescope (NOT) on the island of La Palma for monitoring mainly four well known gravitational lens systems: QSO0142-100, QSO0957+561, QSO1413+117 and QSO2237+0305 (The Einstein Cross).

Here we report results from the monitoring of the Einstein Cross. During the four year period the Monitor Program was active (1990-1993) we gathered 55 high quality observations of this well known lens system. In addition we have collected 32 more observations in 1994 and a further four so far in 1995, a total of 91 new observations of the Einstein Cross.

The observations have all been made with the 2.54 meter Nordic Optical Telescope. For most of the observations the Stockholm CCD camera was used. This camera applies a Tektronix $512^{2}$ chip giving a resolution of $0.2 \times 0.2$ ". From 1994 most observations were made with the new Brorfelde CCD camera whose thinned Tektronix $1024^{2}$ gives an improved resolution of $0.176 \times 0.176$ ", as well as better noise characteristics and quantum efficiency. 


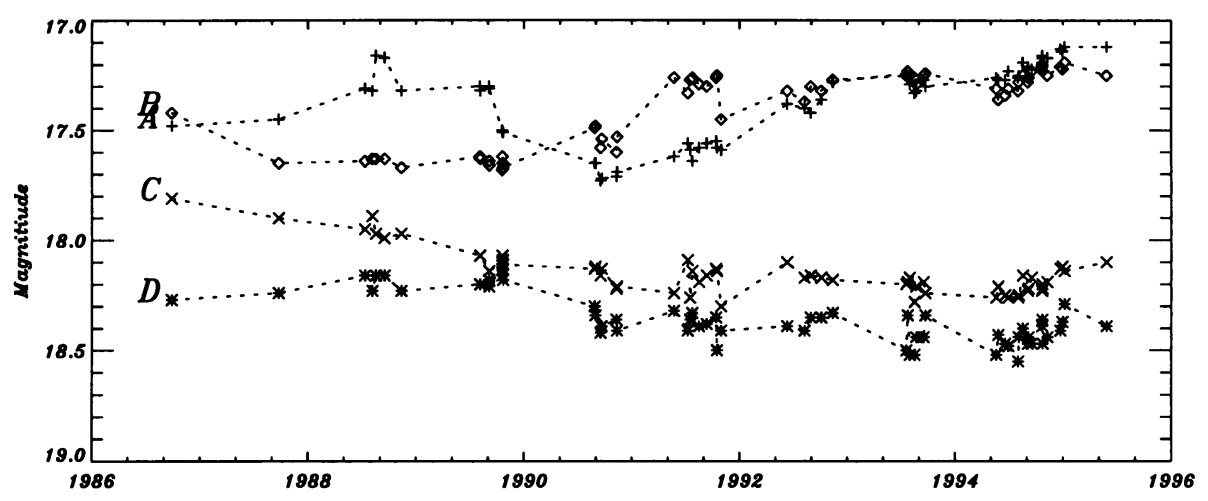

Figure 1. R-band observations

For the preprocessing of the images the IRAF/ccdred package was used. A processing tool developed especially for precise separation of the close components of the Einstein Cross was used to compute the magnitudes of the four quasar images. The program, which has been named XECClean, applies an interactive CLEAN processing algorithm using four point sources and an analytical galaxy model. A Menu/Button X-windows interface has been implemented using IDL, making XECClean easy to use and able to produce good results in less than five minutes. A complete description of XECClean is given in Østensen's thesis (1994) (available from the author).

The most complete light-curves published until now are in the article by Corrigan et al. (1991), where they presented the results from all the direct image CCD data, of sufficient quality, available at that time. Their best sampled light-curve (R-band) includes 15 data points.

Using the Monitor Program observations to extend these light-curves we get curves like the one shown in Figure 1. Due to the limited space available we show only the best sampled light-curve, namely the $\mathrm{R}$ band data. Figure 1 shows the measured magnitudes for the four components. Data points prior to 1990 are all from Corrigan et al. (1991).

The resulting light-curves exhibit several micro-lensing features, and variations on time-scales of several years are found in all four images. For complete tables of magnitudes and error estimates, as well as further interpretations of the features revealed in the light-curves, the reader is referred to Østensen et al. (1995)

\section{References}

Corrigan, R.T., Irwin, M.J., Arnaud, J., et al., 1991, AJ, 102, 34

Østensen, R., 1994, Cand. Scient. Thesis, University of Troms $\varnothing$

Østensen, R., Refsdal, S., Stabell, R., Teuber, J., et al., 1995, A\&A, in press 